was found. It was curious, too, how the secondary tumours in the lung and sternum kept to the original type, while those in the liver and skull showed no trace of colloid degeneration. We have not been able to find record of any similar affection of bone like that in the skull by a new growth in which at no time was there any increase of tissue or enlargement of the bone.

Our thanks are due to Dr. Drew and Dr. Curtis, surgical registrars of University College Hospital, and Mr. R. Johnson, who have given much help in the early history of the case.

\section{A SUGGESTION FOR TREATING THE ATTACHED BORDER IN SIMPLE SUTURE OF THE INTESTINE.}

\section{BY J. ERNEST FRAZER, F.R.C.S. ENG.}

THE undoubted fact that serous membranes form adhesions more rapidly and firmly to tissues which are not serous than to those which are was first brought forward in print, I believe, by the late Mr. Greig Smith, but I only know of one suggestion for intestinal suture which makes use of this fact-I refer to Dr. Roger's ingenious method. ${ }^{1}$ It seems to me, however, that in carrying out this method there is some possible danger of interfering with the blood-supply of the muscular coat, from which the serous cuff is stripped and turned back, and of that serous coat itself, and perhaps an equally advantageous result could be obtained by ordinary Lembert sutures placed in one segment of the bowel, nearer to the divided edge than in the other segment. My motive in this communication, however, is not to criticise or advance methods of suture for the general circumference of the divided intestine, but to make a suggestion for the treatment of the attached mesenteric border of the out, based on what is, I think, a new principle, and depending for its success on the quick adhesion between peritoneal and non-peritoneal surfaces; nor is it my intention to discuss the reasons why, where, and how various appliances should or should not be used instead of simple stitching without appliances, for not only have these matters been dealt with by many who speak with great authority, but they do not bear at all upon my subject. The difficulty and loss of time and subsequent danger which are met with in joining the mesenteric intestinal attachments do not arise when the ordinary mechanical appliances such as the various tubes and buttons are used, but are always present when the gut is being simply stitched. The cause of this drawback to simple suture is, of course, the length of time which must elapse before two non-serous surfaces adhere with any firmness. This is too great to be covered by the protective time afforded by the prolapse of the mucous membrane and the exudation and swelling in the line of division and stitching. No matter how carefully and ingeniously the peritoneum is folded in and stitched over the triangular space at this border there must be some pointperhaps not more than a mere point-in each segment where there is no peritoneal covering, and when the mesenteric attachments are brought together these points may perhaps be opposed to one another, thus leaving the danger still existing, though minimised. This danger would not exist if these non-serous points or surfaces were opposed to serous membrane, when, instead of being the weakest points in the sutured circumference, the mesenteric junctions might be expected to be amongst the strongest.

The suggestion which I venture to put forward for accomplishing this object has the merit of simplicity, and, being of the nature of a principle, the further one that it can be adopted while the operator uses any form of stitch which may be captivating his fancy at the moment. I intend to describe it as used with ordinary interrupted Lembert sutures and to say a few words afterwards about its use with one or two other varieties. It consists essentially in opposite rotation for a little distance, on the longitudinal axis, of each segment of the gut, ore forwards and the other backwards, so that the plane of the mesentery of one segment lies a little behind that of the other; when the two segments are fixed in this position, the tissues in the triangular intervals are opposed to the opposite peritoneal coat, to which they can adhere quickly and firmly, and when this portion of the circumference is united the remainder, with the divided mesentery, can be sutured in the usual way. The accompanying diagram which is intended to show the sutures passed (the mesentery for the sake of clearness being represented as cut away near its attachment) explains what I mean. It will be observed that three stitches are shown between the separated mesenteries, but the number is of course quite optional. I will return to this point later.

The blood-vessels of the gut differ in their general relations at the attached border and in the rest of the circumference. The ultimate branches of the last anastomotic mesenteric loops lie at first between the muscular coat and the serous membrane which has here left the muscular coat but has not yet met its other layer to form the general mesentery. For this reason the stitches which (at $A$ and $\mathrm{B}$ in the diagram) pierce the peritoneum in this position must only take up the serous coat, while in the other segment ( $C$ and $D$ ) they can take some

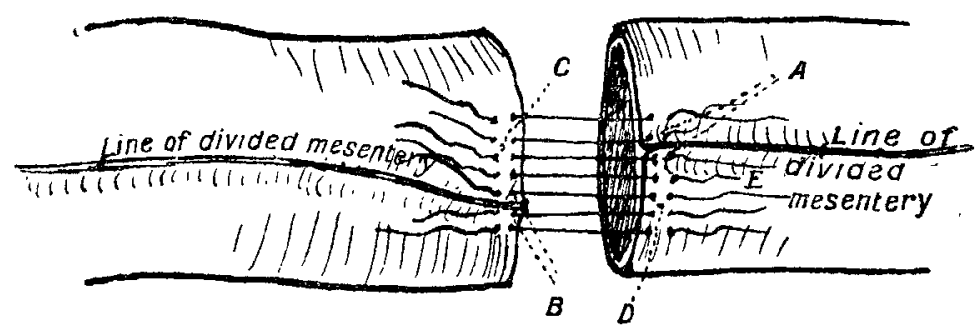

of the muscular layer as well. These ultimate arteries, instead of piercing the muscular floor of the triangular interval, may run for a little distance under the serous coat before sinking into position, so that it would perhaps be better, at least theoretically, if the middle suture (E) only involved the serous coat on both sides and the outermost sutures did the same at their mesenteric sides. If thought necessary the muscular coat exposed in the triangular space could be stitched directly to the opposing peritoneum by a separate suture; this, I think, should certainly be done in fat subjects and preferably in all. When about to place these sutures as much mesentery as possible should be withdrawn through the incision, so that plenty of "slack" is obtained to facilitate their introduction. The segment of intestine lying near the operator's right hand is that the mesentery of which should assume the posterior plane. The first stitch is passed through the anterior layer of the right mesentery where it rises from the muscular coat in the usual Lembert manner, except that it only involves the serous coat; after this the needle is introduced into the other segment at a proper distance behind the left mesentery, involving the serous and muscular coats and leaving the suture in position. The second suture is then passed in front of the first (only involving, if thought necessary, for the reasons already given, the serous coats). The third stitch is then placed through both coats of the right segment and through the rising posterior layer of the left mesentery, thus involving the serous layer only. The fourth stitch includes the two coats of the right segment and the serous coat of the rising anterior layer of the left mesentery. The fifth stitch is in front of this. When these sutures are tied the sixth one can be placed behind the right mesentery and the suturing of the remaining circumference commenced.

For the sake of simplicity I have followed the diagram in this description of the placing of the stitches which carry out the principle, but practically it will be found, especially by those who place their sutures very close together, that each rising mesenteric layer will generally take two stitches, so that with a centre stitch five sutures will be required between the mesenteries. It is well, I think, to assume that a suture should not pass directly from the anterior layer of one mesentery to the posterior layer of the other, so that two inter-mesenteric sutures would be the smallest number, thus giving a range from two upwards, the number depending on the opinion and methods of the operator; from three to five would be the usual number employed, not counting the stitches through the floor of the triangle.

The sutures can, of course, be placed in any way found most convenient by the operator ; I have found no difficulty in doing this or in tying them, the time required being little. if at all, longer than that required in placing an equal number of Lembert sutures in the general circumference, greater facility being given by turning the left segment 
upwards and backwards so that the points of future approximation come together. I think, too, that some help is given by tying each stitch as it is passed, leaving the ends long for holding up the gut wall; but the stitch passing through the floor of the space is more easily placed before those immediately beside it are tied and tied afterwards. The tendency is usually to pass the first stitch further behind the left mesentery than originally intended, but this is a matter of no practical importance, as it only means an addition to the number of ifter-mesenteric stitches.

If Mansell's method be preferred the separation of the two mesenteries is easily effected by passing the first guiding suture through the walls of the segment in front of one mesentery and behind the other; the second thread is passed at the opposite point to the first and the operation proceeds as usual. It must be borne in mind that the longitudinal incision is not in a line with the second stitch, but is opposite the mesentery of the segment in which it is made. I have also tried the proposed method of fixing the attached borders, using the short continuous Lembert suture as recommended by Robinson. This plan certainly shortens the time spent over the operation and seems to give a very good result, at least in the dead bowel. As the base of the triangular space is very little under one-third of an inch across and the rising layers do not come together for nearly three-quarters of an inch, the sutures involving these layers on either side are necessarily when first placed further apart than at their insertions in the other segment, but owing to the looseness of the mesenteric layers at this point they turn in and fit accurately when tied.

As has been indicated, the great theoretical advantage which lies in the principle suggested is in the fact that at every point in the sutured circumference there is peritoneum on one or both sides, thus allowing no more danger at the attached borders than at any other part of the divided edges. To this I venture to add the practical advantages of adaptability to any form of stitching preferred by the operator and rapidity of execution, for not only are the stitches easily applied, but the time spent in securing the mesenteric regions is at the same moment spent in suturing a corresponding length of the whole circumference.

West Dulwich, S.E.

\section{A CONTRIBUTION TO THE ANATOMY OF THE PERITONEUM.}

BY FRED. J. SMITH, M.D. OXON., F.R.C.P. LOND., SENIOR PATHOLOGIST TO THE LONDON HOSPITAI;

C. MANSELL MOULLIN, F.R.C.S. FNG., SURGEON TO THE LONDON HOSPITAL;

$A N D$

ARTHUR KEITH, M.D. ABERD., F.R.C.S. ENG.,

SEAIOR DEMONSTRATOR OF ANATOMY TO THE LONDON HOSPITAL MEDICAL COLLEGE.

THat the two following extraordinarily rare cases should come under observation within six weeks of one another in the post-mortem room of one hospital is an illustration of the manner in which pathological curiosities seem to occur in groups.

CASE 1.-A man was admitted to the London Hospital on Dec. 2nd, 1898, with signs of acute peritonitis. Laparotomy was speedily followed by death which occurred on Dec. 5th. On opening the abdomen post-mortem purulent peritonitis was found with a feculent smell suggesting perforation of the gut. Turning over the intestines in search of the wound Dr. F. J. Smith became aware of a dense, hard, fibrous ring, through which a mass of small intestine seemed to have passed. More careful examination led to the localisation of this ring. It was situated just at the lower end of the ascending colon, nearly opposite the cæcum, an inch or so from its inner border. The ring itself was very hard and definite. It was about two inches in diameter with a complete and uniform circumference. Before the herniated gut could be withdrawn the ring required to be freely incised; as soon as this incision was made the operator was able to withdraw an incarcerated hernia of small intestine of about the size of a large orange. There were no signs of strangulation at all, but a perforation was found at the neck of the mass, due apparently to attrition against the hard unyielding ring of the sac. This was the cause of the peritonitis and cleared up the case from a clinical point of view. The ring and sac were cut out and aremow in the museum of the London Hospital.

CASE 2.-A man, aged 20 years, was admitted into the London Hospital for fracture of the spine on Jan. 11th, 1899. He was in perfect health till the time of the accident and the abdominal condition was purely incidental with no bearing or influence upon his case. The patient died on the same day. On opening the abdomen and lifting the omentum it at once became apparent that there was an unusual condition of things to be investigated. The small intestines, instead of being loose and moveable upon one another, were found to be enclosed in a large sac with a thin peritoneal covering in front of them so that the coils could not be actually touched or individually separated. The ascending colon was situated a little to the left of the mid-abdominal line and had almost the whole of the small intestine lying to the right of it. Investigation showed that this peculiar relationship was due to the fact that the small gut - almost the whole of it - had slipped through a bole at the lower and inner margin of the ascending colon situated in precisely the same spot as in Case 1 . The margin or ring of the cavity thus formed was very different in condition in this case from that of Case 1. It was detinite and defined enough but very thin, membranous, and lax, and had exerted no strangling or incarcerating influence on the contents of the sac; it was so thin and so easily continuous with the mesentery-i.e., without definite change of feel or struc

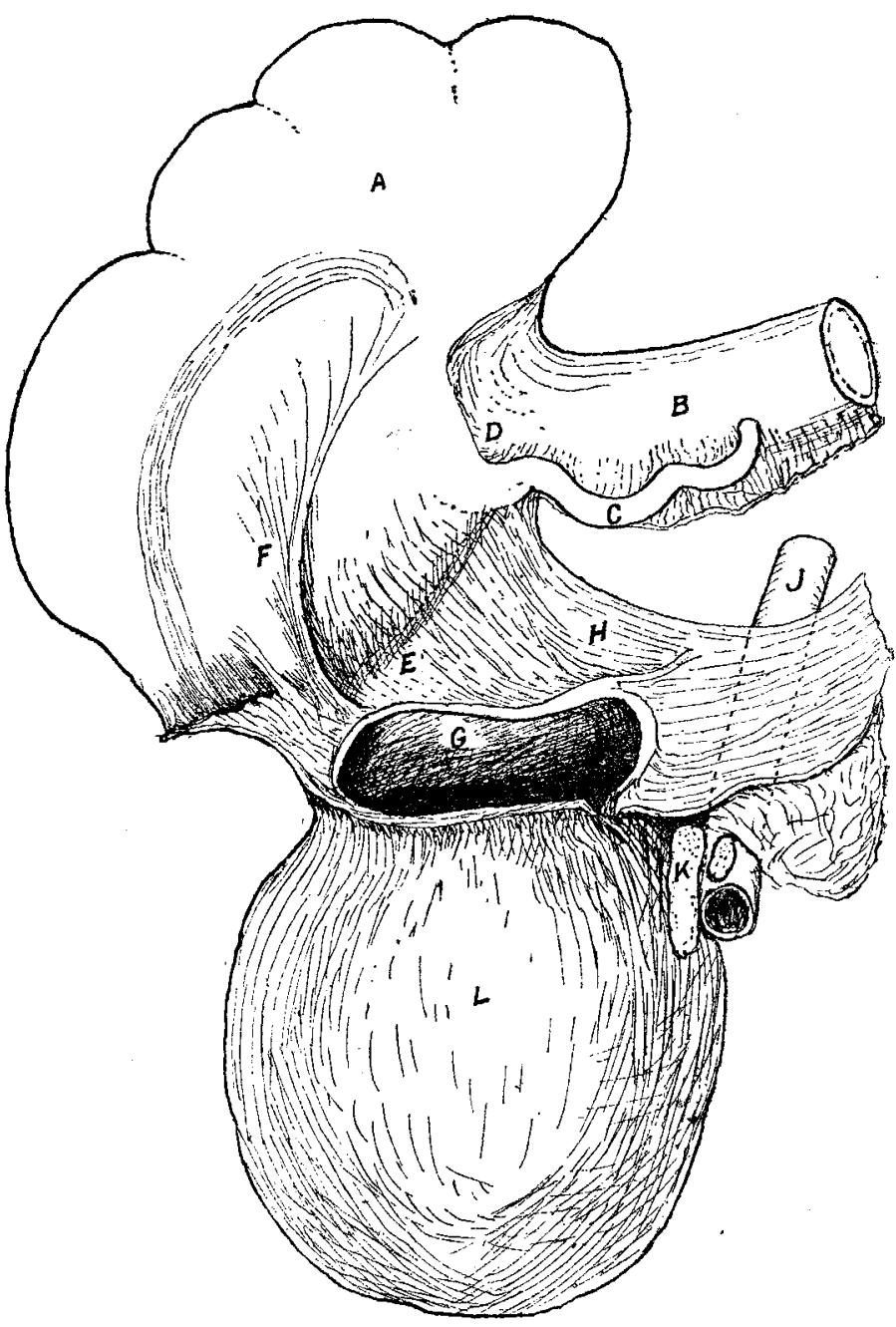

Specimen removed from Case 1. A, Cæcum thrown upwarls. B, Ileum, $C_{2}$ Appendix. D, Ileo-cacal fossa. $\mathbf{E}$ Retro creal fossa , A A scending mesocolon. Mouth of the cacal fossa. $F$, Ascending mesocolon. G, Mouth of the iliac artery projected within the pelvis towards I, Hernial sac

ture-as to be incapable of removal. After passing through this ring the small intestine had insinuated itself upwards and downwards along the whole length of the ascendin colon and cæcum and appendix in the space ordinarily termed retro-peritoneal, removing them entirely from their usual position and relations on the right of the abdominal cavity and lifting them bodily to or beyond the middle line. The ring and tissues of the sac were so thin that Dr. F.J. Smith is unable to state whether between the herniated intestine and the omentum there were one or two layers of 